\title{
Achados oculares em pacientes com mucopolissacaridoses
}

\author{
Ocular findings in patients with mucopolysaccharidosis
}

Flávia Silva Villas-Bôas ${ }^{1}$, Dilson José Fernandes Filho², Angelina Xavier Acosta ${ }^{2}$

\section{RESUMO}

Objetivo: $\mathrm{O}$ objetivo deste estudo foi determinar a prevalência e gravidade das complicações oculares em pacientes com mucopolissacaridoses (MPS).

Métodos: Vinte e nove pacientes com diagnóstico de mucopolissacaridoses foram estudados. Foram avaliados: idade, sexo, acuidade visual, presença de estrabismo, erros refrativos, exame de fundo de olho, pressão intraocular, espessura corneal central e ultrassonografia ocular.

Resultados: Foram avaliados três pacientes com MPS I (12\%), 11 pacientes com MPS II (37,9\%), um paciente com MPS III (3,4\%) e 14 pacientes com MPS VI (48,3\%). A média de idade foi de 9,5 anos (DP 5,5). Observou-se hipermetropia em 88,5\% (23 pacientes) e astigmatismo em 51,7\% (15 pacientes). A média da acuidade visual corrigida foi de 0,45 logMAR (DP 0,68). A média do equivalente esférico foi +3,57 D (DP 2,46) e da pressão intraocular foi $17 \mathrm{mmHg}$ (DP 3,9). Os achados mais comuns foram: espessamento palpebral 24,1\% (7 pacientes); opacidade da córnea, 55,2\% dos casos (16 pacientes); atrofia do nervo óptico, 23,1\% (6 pacientes); dobras radiais na retina $24 \%$ ( 7 pacientes). 0 fundo de olho não foi examinado em 3 pacientes devido à opacidade de córnea. A média da espessura do complexo esclera-retinacoroide (ERC) medida por ultrassom foi de 1,78 mm (DP 0,51).

Conclusão: Os achados oftalmológicos mais proeminentes foram espessamento palpebral, diminuição da acuidade visual, hipermetropia moderada, opacidade da córnea, dobras radiais na retina perimacular e atrofia do nervo óptico.

Descritores: Mucopolissacaridose/diagnóstico; Mucopolissacaridose/ultrassonografia; Beta-galactosidase/metabolismo; Opacidade da córnea; Oftalmopatias/etiologia; Esclera/patologia; Iris/patologia; Córnea/patologia; Atrofia óptica; Nervo óptico; Acuidade visual

\section{ABSTRACT}

Purpose: The objective of thisstudy was to determinetheprevalenceand severity ofocular complications in patients with mucopolysaccharidosis (MPS)

Methods: Twenty-nine patients with diagnosis of mucopolysaccharidosis were studied. Age, gender, visual acuity, presence of strabismus, refractive error, fundus examination, intraocular pressure, central corneal thickness and ocular echography were assessed for each individual.

Results: There were three patients with MPS I (12\%), eleven patients with MPS II (37.9\%), one patient with MPS III (3.4\%) and fourteen patients with MPS VI (48.3\%). Mean age was 9.5 years (ranged from 1.2 to 20 years, DP 5.5). Refraction was available in 26 patients, from which $88.5 \%$ (23 patients) were hyperopic, and 53.8\% (14 patients) presented astigmatism. Best corrected visual acuity was available in 18 patients and the mean was $0.45 \log M A R$ (DP 0.68). The mean spherical equivalent was +3.57 D (SD 2.46) and intraocular pressure was $17 \mathrm{mmHg}$ (SD 3.9). The most common findings were: eyelid thickening in $24.1 \%$ (7 patients); corneal opacity in $55.2 \%$ of cases (16 patients); optic nerve atrophy in $23.1 \%$ ( 6 patients); and radial folds in the retina in 24\% (7 patients). The fundus was examined in 26 out of 29 patients because corneal opacity avoided the exam in 3 of them. The average thickness of the complex sclera-retina-choroid (SRC was $1.78 \mathrm{~mm}$ (SD 0.51).

Conclusion: The most prominent ophthalmologic findings were eyelid thickening, decreased visual acuity, high hyperopia, corneal opacity, perimacular radial folds in the retina and optic nerve atrophy.

Keywords:Mucopolysaccharidosis/diagnosis; Mucopolysaccharidosis/ultrasonography; Beta-galactosidase/metabolism; Cornealopacity; Eyediseases/etiology; Sclera/pathology; Iris/pathology; Cornea/pathology; Optic atrophy; Optic nerve; Visual acuity

\section{INTRODUÇÃO}

As mucopolissacaridoses (MPS) são um grupo de doenças caracterizadas pelo acúmulo de glicosaminoglicanos (GAG) em vários tecidos, devido à deficiência de enzimas envolvidas na degradação de GAG, também chamados mucopolissacarídeos ${ }^{(1)}$. As MPS são divididas em tipos que abrangem um espectro amplo de fenótipos, os quais vão desde distúrbios fatais nos primeiros meses de vida até aqueles compatíveis com uma qualidade de vida razoável ${ }^{(2)}$. O padrão de herança é autossômico recessivo, sendo que a MPS II é recessiva e ligada ao $X^{(3)}$. A incidência das MPS é descrita como sendo de três a cinco casos a cada 100.000 nascidos vivos e a MPS I é a ocorrência mais frequente ${ }^{(4,5)}$.
As complicações oftálmicas são bastante frequentes em pacientes com MPS(6,7). Opacidade da córnea, hipertensão ocular e glaucoma, retinopatia e neuropatia estão entre as alterações oculares mais descritas. O tratamento oftalmológico de muitos pacientes com MPS tem sido tradicionalmente conservador por causa da baixa espectativa de vida e comprometimento intelectual de alguns pacientes. Entretanto, os tratamentos modernos, incluindo a terapia de reposição enzimática (TRE) para MPS I, II e VI, podem resultar em uma maior sobrevida e melhor qualidade de vida para estes pacientes, o que aumenta o interesse no diagnóstico precoce das alterações sistêmicas geradas pela MPS(8-10).

Este trabalho visa contribuir para o conhecimento das alterações oftalmológicas em pacientes com MPS, já que há poucos es-

Submitted for publication: May 12, 2011

Accepted for publication: October 26, 2011

Study carried out at the IBOPC - Instituto Brasileiro de Oftalmologia e Prevenção da Cegueira.

Physician, Instituto Brasileiro de Oftalmologia e Prevenção da Cegueira - IBOPC - Mestrado, Fundação

Bahiana para Desenvolvimento das Ciências, Salvador (BA), Brazil.

2 Physician, Professor, Universidade Federal da Bahia - UFBA - Salvador (BA), Brazil.

Funding: No specific financial support was available for this study.

Disclosure of potential conflicts of interest: F.S.Villas-Bôas, None; D.J.Fernandes Filho, None; A.X.Acosta, None.

Correspondence address: Flávia Silva Villas-Bôas. Avenida Princesa Leopoldina, 288 - Apto. 901 Ed. Mansão Princesa Leopoldina - Salvador (BA) - 40150-080 - Brazil

E-mail: f-villasboas@bol.com.br

Número do projeto e instituição responsável pelo parecer do Comitê de ética

Comitê de Ética em Pesquisas (CEP) da Escola Baiana de Medicina e Saúde Pública número 73/ 2009, ofício 501/2009. 
tudos que avaliam a doença, incluindo exame oftalmológico completo e ultrassonografia ocular.

\section{MÉTODOS}

Foram examinados no Instituto Brasileiro de Oftalmologia e Prevenção da Cegueira (IBOPC) localizado em Salvador, do ponto de vista oftalmológico, 29 pacientes com diagnóstico de MPS acompanhados no serviço de genética médica no Hospital das Clínicas, em Salvador. Os pacientes ou seus responsáveis responderam um questionário completo com antecedentes familiares, médicos e oftalmológicos do paciente. Foram avaliados: idade dos pacientes no momento do exame, sexo, acuidade visual corrigida, erro refracional, presença de desvios oculares, ectoscopia, biomicroscopia, tonometria, paquimetria, mapeamento de retina e ultrassonografia. A acuidade visual foi realizada utilizando a tabela de visão subnormal e convertida para logMAR. O grau de opacidade da córnea foi subjetivamente graduado (por um único observador) como nenhum (0), leve $(+)$, moderada $(++)$, ou grave $(+++)$ (Figura 1).

A medida da pressão intraocular (PIO) foi realizada pelo tonômetro de Goldmann (Haag-Streit, Koniz, Suíça) e a espessura central corneal foi medida através de paquimetria ultrassônica (Alcon OcuScan RXP Irvine, Califórnia, EUA). A sedação não foi utilizada para o exame e os pacientes que não conseguiram realizar os testes por falta de capacidade cognitiva ou de colaboração foram excluídos da análise correspondente. A ultrassonografia foi realizada com aparelho de ultrassonografia ocular (UltraScan, Alcon, Califórnia, EUA), transdutor de $10 \mathrm{MHz}$ com avaliação do comprimento axial ocular, espessura média da parede ocular, presença de sobrelevação do nervo óptico e/ou papila evidenciável. A parede ocular dos olhos examinados ao ultrassom foi medida a cerca de $4 \mathrm{~mm}$ do nervo óptico nos meridianos das 3, 6, 9 e 12 horas, por convenção, e calculada a média das medidas. $O$ estudo foi realizado em conformidade com os princípios da Declaração de Helsinki e foram obtidos o consentimento informado e a aprovação Comitê de Ética da Faculdade Bahiana de Medicina e Saúde Pública.

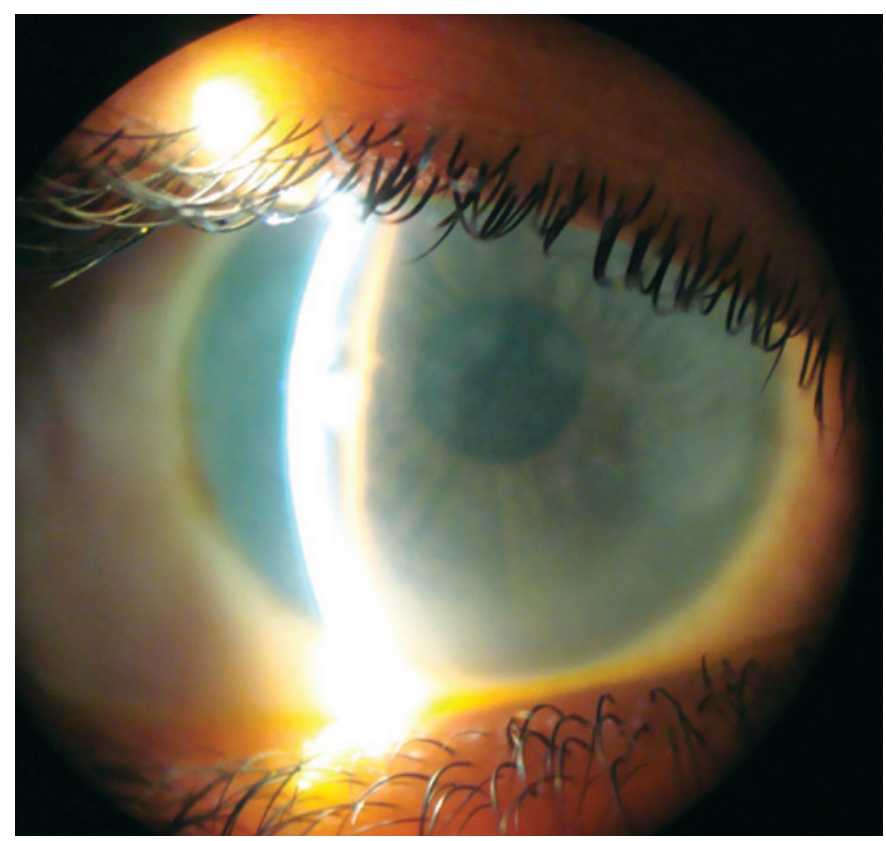

Figura 1. Paciente com mucopolissacaridose tipo VI apresentando opacidade cornea moderada.

\section{RESULTADOS}

\section{Características demográficas e relacionadas À MuCOPOLISSACARIDOSE}

Um total de 29 pacientes foram avaliados com diagnóstico de MPS. Entre eles, 10\% (3 pacientes) apresentavam MPS I, 38\% (11 pacientes) apresentavam MPS II, 4\% (1 paciente) apresentavam MPS III e 48\% (14 pacientes) apresentavam MPS VI. O sexo masculino foi o mais prevalente, perfazendo $82,8 \%$ do grupo total (24 pacientes).

Outros casos na família foram descritos por $62 \%$ dos pacientes (18 pacientes), sendo que o grau de parentesco "irmão" foi o mais frequente, correspondendo a $72,2 \%$ dos casos de consanguinidade (13 pacientes). Dos 29 pacientes, apenas 10\% (3 pacientes) não se encontravam em tratamento com TRE.

A média de idade dos pacientes, no momento do exame, foi de 9,5 anos (DP 5,5). A média de idade referida para o aparecimento dos sintomas foi de 1,5 anos (DP 1,0) e para o diagnóstico foi de 3,5 anos (DP 2,8). A média de idade para o início do tratamento foi de 7,5 anos (DP 4,6).

\section{EXAME OFTALMOLÓgico}

Em todos os pacientes, a ectoscopia foi avaliada e os achados mais frequentes foram espessamento palpebral (Figura 2), exorbitismo, hipertelorismo e epicanto. A distribuição dos mesmos está expressa na tabela 1.

Dos 29 pacientes, 17,2\% (5 pacientes) apresentaram desvios oculares, sendo que 60\% (3 pacientes) apresentaram exotropia e 40\% (2 pacientes), esotropia.

A refração foi possível em 26 pacientes e o erro refracional mais frequente foi a hipermetropia que correspondeu a $79,3 \%$ dos casos (23 pacientes). A miopia esteve presente em 10,3\% dos casos (3 pacientes) e o astigmatismo em 51,7\% (15 pacientes). Na biomicroscopia anterior, os achados mais frequentes foram opacidade da córnea (Figura 1), presente em 55\% dos casos (16 pacientes) e alargamento do limbo superior, $17,2 \%$ dos casos (5 pacientes). A

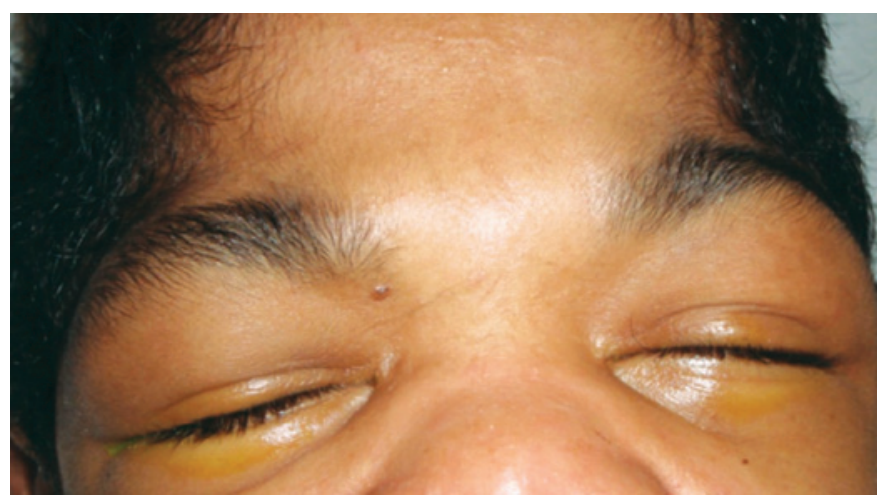

Figura 2. Espessamento da pele das pálpebras em paciente com mucopolissacaridose tipo II.

Tabela 1. Avaliação da ectoscopia

Total $=29$ pacientes

\begin{tabular}{lr}
\hline Sem achados & $51,7 \%(15)$ \\
Espessamento palpebral & $24,1 \%(7)$ \\
Exorbitismo & $13,8 \%(4)$ \\
Hipertelorismo & $6,9 \%(2)$ \\
Epicanto & $3,4 \%(1)$ \\
\hline
\end{tabular}


classificação da opacidade da córnea está na tabela 2. Estreitamento da câmara anterior foi observado em 6,9\% dos casos (2 pacientes) e catarata em 10,3\% dos casos (3 pacientes). Em todos os casos, as cataratas eram nucleares e houve relato do uso de corticosteroides por período de tempo superior a seis meses.

A presença de neuropatia, retinopatia e a classificação dos achados relacionados estão apresentadas nas tabelas 3 e 4 . Alguns pacientes foram excluídos desta análise devido ao grau de opacidade de córnea que impossibilitou o exame do fundo de olho.

As médias dos valores numéricos de acuidade visual corrigida, equivalente esférico, $\mathrm{PIO}$, espessura de córnea central, comprimento axial e espessura do complexo ERC dos olhos dos pacientes com MPS estão expressas na tabela 5.

O equivalente esférico estratificado por faixa etária mostrou uma média de $+1,68$ D (DP 1,2) nos pacientes até 3,4 anos; +6,07 D (DP 1,90) na faixa etária de 3,5 a 06 anos e +3,80 D (DP 2,45) a partir dos 6 anos.

O estudo ultrassonográfico do nervo óptico não mostrou alterações em 72,4\% dos casos (21 pacientes). Em 24,1\% dos pacientes ( 7 pacientes), foi observada sobrelevação do nervo óptico (Figura 3) em pelo menos um olho. Em 3,4\% dos casos (1 paciente) foi vista escavação evidenciável ao ultrassom.

Gráficos comparativos entre os tipos de mucopolissacaridoses com relação aos achados de ectoscopia, grau de opacidade da córnea, achados do fundo de olho e neuropatias estão expressos na figura 4.

\section{DISCUSSÃO}

Este foi o estudo brasileiro com maior número de pacientes que avaliou os achados oftalmológicos das MPS. As características relacionadas à MPS dos pacientes estudados demonstram a distribuição dos tipos de MPS em nosso meio e os atrasos observados no diagnóstico e tratamento da doença. Os achados oftalmológicos mais proemi-

\section{Tabela 2. Grau de opacidade da córnea}

\begin{tabular}{lc}
\hline & Total= 29 pacientes \\
\hline Ausente & $44,8 \%(13)$ \\
Leve & $27,5 \%(8)$ \\
Moderado & $17,2 \%(5)$ \\
Grave & $10,3 \%(3)$ \\
\hline
\end{tabular}

Tabela 3. Presença de neuropatia óptica e classificação dos achados

\begin{tabular}{lc}
\hline & Total $=\mathbf{2 6}$ pacientes \\
\hline Ausente & $61,5 \%(16)$ \\
Atrófica & $23,1 \%(6)$ \\
Escavada & $3,8 \%(1)$ \\
Edematosa & $3,8 \%(1)$ \\
Palidez & $7,7 \%(2)$ \\
\hline
\end{tabular}

Tabela 4. Avaliação da retinopatia e classificação dos achados

\begin{tabular}{lc}
\hline & Total= $\mathbf{2 6}$ pacientes \\
\hline Ausente & $51,7 \%(15)$ \\
Dobras radiais na retina perimacular & $24,0 \%(7)$ \\
Aumento da tortuosidade vascular & $20,6 \%(6)$ \\
Estreitamento arteriolar & $3,4 \%(1)$ \\
Manchas hipocrômicas na retina & $3,4 \%(1)$ \\
\hline
\end{tabular}

nentes foram espessamento palpebral, diminuição da acuidade visual, hipermetropia moderada, opacidade da córnea, dobras radiais na retina perimacular e atrofia do nervo óptico. Ao ultrassom, os achados mais relevantes foram aumento da espessura do complexo esclera-retina-coróide (ERC) e sobrelevação do nervo óptico.

A distribuição dos tipos de MPS neste estudo foi semelhante à descrita por alguns autores ${ }^{(11)}$ em estudo realizado com 113 pacientes com MPS no Brasil, em que foi observada uma prevalência menor de pacientes com MPS I (18 pacientes) e maior dos tipos II e VI (43 e 29 pacientes, respectivamente). Entretanto, mesmo no estudo de Vieira et al. ${ }^{(11)}$, os pacientes com MPS VI constituíram apenas $25,6 \%$ da amostra, sendo que em nosso estudo, eles representaram 48\% (14 pacientes) do grupo total. Isso ocorreu, provavelmente, devido à incidência elevada deste tipo de MPS em determinadas regiões do sertão baiano, em especial na cidade de Monte Santo, como foi descrito em estudo prévio realizado na localidade, fato que ainda necessita de maiores esclarecimentos ${ }^{(11)}$. A alta prevalência do sexo masculino em nossa amostra, provavelmente, foi influenciada pelo alto número de pacientes com MPS II (11 pacientes) que tem herança recessiva ligada ao $X$.

Observou-se uma percepção mais rápida da doença por parte dos familiares, neste estudo, em comparação com a literatura ${ }^{(11)}$. As razões para este achado provavelmente estão relacionadas à presença de grande número de outros casos na família dentre os pacientes estudados, o que aumenta a atenção dos familiares e profissionais assistentes para os sinais precoces da doença. Apesar disso, ainda há um atraso significativo entre a percepção dos sintomas, diagnóstico e início do tratamento. Em estudo realizado nos anos de 2005 e 2006 numa coorte de centros brasileiros, foi observado um atraso médio de quatro anos e dez meses entre o aparecimento dos sintomas e o diagnóstico ${ }^{(11)}$. O diagnóstico mais precoce observado no presente estudo deve-se, provavelmente, ao aumento do interesse nos conhecimentos sobre a MPS nos últimos anos, após o surgimento de tratamentos mais modernos, além da busca ativa de casos realizada nas regiões do sertão baiano onde foi observada maior prevalência da doença. Infelizmente, mesmo com essas iniciativas, o início do tratamento ainda se encontra bastante defasado em relação ao diagnóstico, provavelmente por conta da existência de poucos centros de tratamento, além das dificuldades de acesso e transporte dos pacientes. Este atraso é crucial para a sobrevivência dos pacientes por tratar-se de uma doença de depósito em que o comprometimento de vários órgãos é progressivo. Neste estudo, 90\% dos pacientes (26 pacientes) estavam realizando a TRE. A TRE tem criado uma nova perspectiva para os pacientes com MPS e já está disponível para as MPS I, II e VI. Devido ao desenho do presente estudo, não foi possível avaliar os efeitos da TRE nos achados oftalmológicos, porém permanece como uma perspectiva em acompanhamentos posteriores.

Dentre os achados oftalmológicos mais frequentes, pode-se observar na ectoscopia, o espessamento palpebral. Devido à fisiopatologia da doença, a causa mais provável para este achado é o espessamento da pele das pálpebras devido ao acúmulo de GAG nos tecidos conectivos. Outro achado frequente na ectoscopia foi o exorbitismo. A protrusão dos globos oculares foi descrito como exorbitismo neste estudo, e não como proptose, como foi observado na maior parte dos estudos anteriores ${ }^{(1,6,7)}$, por considerarmos que o componente de protrusão do globo ocular se deve, essencialmente, à conformação rasa das órbitas e não ao aumento de volume no espaço intraconal.

Foi observada uma diminuição importante da média de acuidade visual dos pacientes com MPS, fato de grande relevância, devido à baixa idade dos pacientes e à grande repercussão que este achado representa na qualidade de vida dos mesmos. Dentre os fatores relacionados à diminuição da visão em pacientes com MPS, a opacidade de córnea representa, certamente, um papel importante. Entretanto, a opacidade corneal não é o único fator 
Tabela 5. Médias de acuidade visual corrigida, equivalente esférico, pressão intraocular, espessura da córnea central, comprimento axial e espessura do complexo esclera-retina-coróide

\begin{tabular}{|c|c|c|c|c|c|c|}
\hline Tipo de MPS & $\begin{array}{l}\text { Acuidade visual } \\
\text { corrigida } \\
\text { (logMAR) }\end{array}$ & $\begin{array}{l}\text { Equivalente } \\
\text { esférico } \\
\text { (dioptrias) }\end{array}$ & $\begin{array}{c}\text { Pressão } \\
\text { intraocular } \\
(\mathrm{mmHg})\end{array}$ & $\begin{array}{l}\text { Espessura da } \\
\text { córnea central } \\
(\mu)\end{array}$ & $\begin{array}{l}\text { Comprimento } \\
\text { axial } \\
\text { (mm) }\end{array}$ & $\begin{array}{c}\text { Espessura média do } \\
\text { complexo ERC } \\
(\mathrm{mm})\end{array}$ \\
\hline I $(n=6)$ & $0,11 \pm 0,13 \quad(n=4)$ & $+7,88 \pm 2,18 \quad(n=4)$ & $12,5 \pm 2,9 \quad(n=4)$ & $526,5 \pm 0,2$ & $22,8 \pm 0,6 \quad(n=6)$ & $1,70 \pm 0,30(n=6)$ \\
\hline II $(n=22)$ & $0,02 \pm 0,04 \quad(n=14)$ & $+2,07 \pm 1,28 \quad(n=18)$ & $17,0 \pm 3,2 \quad(n=20)$ & $494,7 \pm 29,2 \quad(n=20)$ & $24,8 \pm 1,24(n=22)$ & $1,95 \pm 0,48(n=22)$ \\
\hline III $(n=2)$ & Não informada & $+0,25 \quad(n=2)$ & $13,0 \pm 0,0 \quad(n=2)$ & $510,5 \pm 12,0 \quad(n=2)$ & $23,8 \pm 0,4 \quad(n=2)$ & $1,05 \pm 0,21 \quad(n=2)$ \\
\hline$V I(n=28)$ & $0,94 \pm 0,75 \quad(n=28)$ & $+4,16 \pm 2,11 \quad(n=28)$ & $18,5 \pm 4,0 \quad(n=18)$ & $644,1 \pm 101,5(n=18)$ & $23,2 \pm 1,3 \quad(n=28)$ & $1,71 \pm 0,53 \quad(n=28)$ \\
\hline Total $(n=58)$ & $0,45 \pm 0,68 \quad(n=34)$ & $+3,57 \pm 2,46 \quad(n=52)$ & $17,0 \pm 3,8 \quad(n=44)$ & $559,4 \pm 99,8 \quad(n=44)$ & $23,8 \pm 1,5 \quad(n=58)$ & $1,78 \pm 0,51 \quad(n=58)$ \\
\hline
\end{tabular}

Os valores apresentados foram obtidos através da média dos valores numéricos dos olhos dos pacientes que conseguiram realizar as medidas. O " $\mathrm{n}$ ” representa o número de olhos em cada análise MPS= mucopolissacaridose; $E R C=$ esclera-retina-coroide

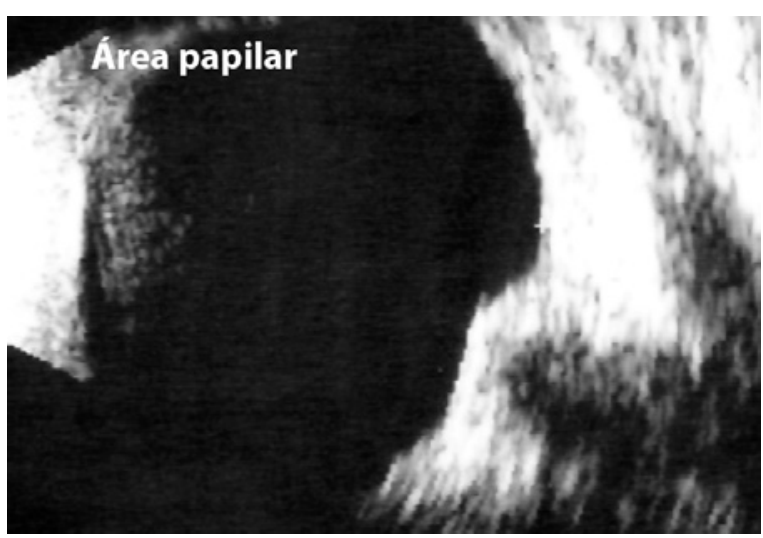

Figura 3. Imagem ultrassonográfica de sobrelevação do nervo óptico em paciente com mucopolissacaridose tipo VI.

envolvido na baixa visão nos pacientes com MPS e é necessária uma análise minuciosa antes de decidir por um transplante de córnea, já que qualquer procedimento cirúrgico nestes pacientes deve ter seu benefício claramente estabelecido pelos riscos cirúrgicos e anestésicos envolvidos. Erros refracionais com dioptrias altas foram frequentes nos pacientes examinados, especialmente a hipermetropia, sendo que a média do equivalente esférico dos pacientes com MPS I chegou a +7,87 dioptrias. As razões atribuídas para esse achado foram a rigidez aumentada da córnea e esclera, o aplanamento da curvatura corneal e o reduzido poder refrativo da córnea, tanto pelo acúmulo de GAG quanto pela diminuição do comprimento axial devido ao espessamento escleral ${ }^{(13,14)}$. Sabe-se que altos erros refracionais não corrigidos em crianças em faixa etária de desenvolvimento da visão podem gerar déficits visuais permanentes. É importante ressaltar que a refração estática de crianças na faixa etária precoce, mostra, normalmente uma hipermetropia latente que, após o crescimento ocular, tenderá ao desaparecimento ${ }^{(15)}$. Entretanto, a hipermetropia em crianças com MPS não apresentou essa característica pois, na faixa etária dos 3,5 anos, quando a hipermetropia normalmente se encontra em cerca de +1,75 D com tendência à diminuição até a faixa etária dos 6 anos; nos pacientes com MPS, o erro refracional apresentou uma média de +6,07 D (DP $1,90)$ e, a partir dos 6 anos, mostrou uma média também mais alta que o esperado(15). Este achado alerta à necessidade da correção precoce do erro refracional para evitar o aparecimento de ambliopia nestes pacientes.

A opacidade da córnea é o achado oftalmológico relacionado à MPS mais estudado. Sabe-se que a opacidade da córnea se mostrou mais grave em pacientes com MPS VI e inexistente em pacientes com MPS II, o que já havia sido observado em outros estudos ${ }^{(6)}$. Este achado parece ter relação com o tamanho dos vacúolos intraestro- mais e com o arranjo das fibrilas colágenas na córnea que diferem entre os tipos de MPS, além do fato de que a deposição de GAG anormal no estroma corneal dos pacientes com MPS II é mínima, embora seja observada grande concentração no endotélio e em estruturas epiteliais da íris, corpo ciliar e esclera ${ }^{(16,17)}$. O alargamento do limbo superior que esteve presente em 5 pacientes. Estima-se que este achado pode estar relacionado à vascularização periférica da córnea devido à exposição corneal associada ao exorbitismo ou pode ser consequência de edema corneal crônico devido à PIO elevada(18). Estreitamento da câmara anterior foi observado em ambos os olhos de um paciente com MPS I e um paciente com MPS VI. A literatura descreve alguns casos de glaucoma agudo em pacientes com MPS $I^{(19,20)}$, entretanto os pacientes do presente estudo não apresentaram sinais ou sintomas de fechamento angular.

A alteração mais frequente no fundo de olho foram dobras radiais da retina na região perimacular que ocorreram em $24 \%$ dos pacientes. Já havia sido relatada a ocorrência de dobras de coroide, provavelmente em decorrência da alta hipermetropia(1). Entretanto, com relação aos olhos examinados no presente estudo, o aspecto das dobras de retina foi mais compatível com o de estrias na membrana limitante interna da retina, e a descrição de um achado semelhante foi observada em um relato de caso de paciente com MPS II em que essas dobras radiais da retina foram comparadas às observadas em casos de retinosquise juvenil ligada ao $X^{(21)}$. A repercussão desta alteração na visão dos pacientes ainda não se encontra estabelecida, o que reitera a importância de uma avaliação mais detalhada deste achado, com exames específicos como tomografia de coerência óptica (OCT) e eletrorretinograma (ERG).

O exame de fundo de olho mostrou alta frequência de atrofia do nervo óptico nos pacientes com MPS, o que é bastante preocupante, já que representa uma perda irreversível da visão. Embora a hipertensão intracraniana seja um fator de grande importância para estes casos, o aumento da espessura do complexo ERC e alterações funcionais da retina decorrentes do acúmulo de GAG podem também estar relacionados com este achado. A média da espessura do complexo ERC esteve, de fato, bastante aumentada nos olhos avaliados nesta população e se constitui num aspecto relevante para futuros estudos que avaliem preditores para o edema e atrofia do nervo óptico em pacientes com MPS.

\section{CONCLUSÃO}

Algumas limitações foram observadas neste estudo: a ausência de estudo eletrorretinográfico pode ter subestimado a ocorrência de retinopatia; a diferença de idade entre os pacientes determina um tempo de evolução da doença distinto, o que representa uma limitação na comparação entre os mesmos.

Este estudo mostrou fatores importantes para a clínica oftalmológica dos pacientes com MPS como a correção adequada do erro refracional e a solicitação de avaliação ultrassonográfica especial- 
Ectoscopia

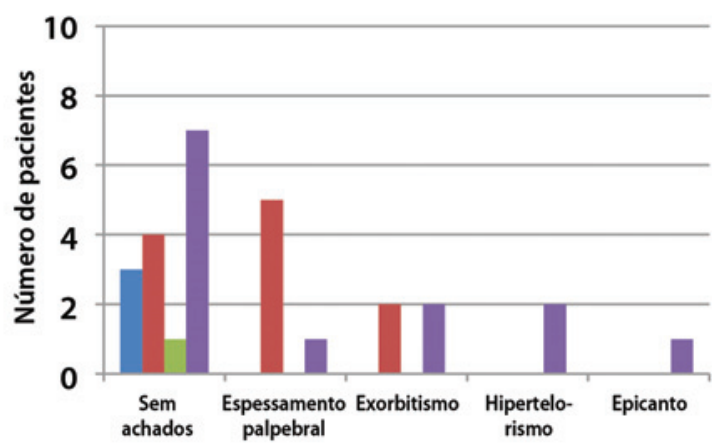

Neuropatia óptica

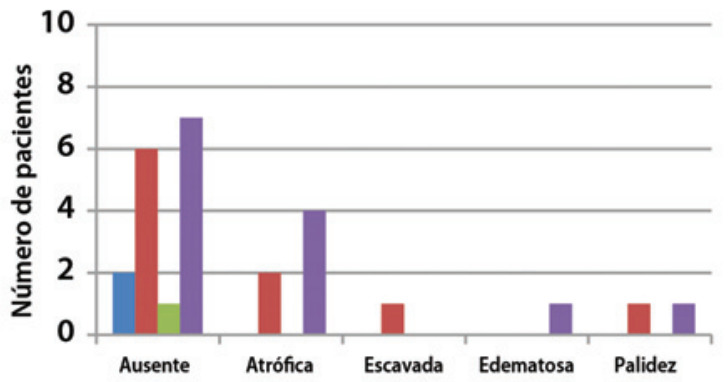

Grau de opacidade da córnea

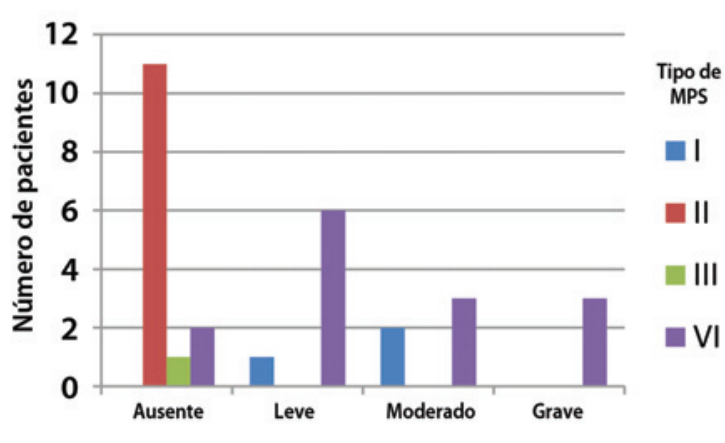

Retinopatia

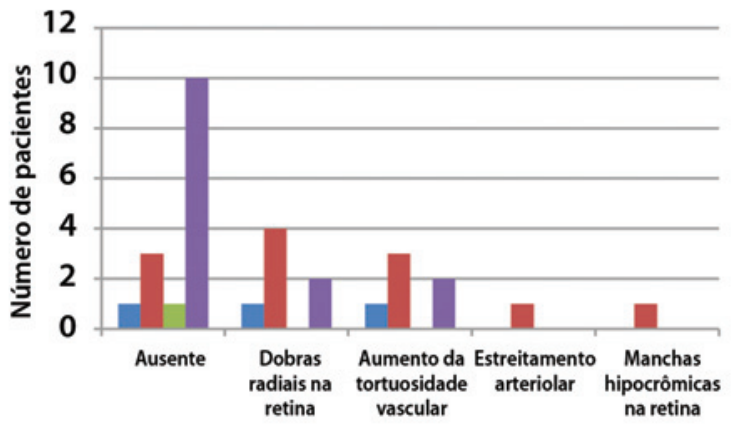

Figura 4. Gráficos comparativos entre os tipos de mucopolissacaridoses (MPS) nos achados de ectoscopia, grau de opacidade da córnea, neuropatia óptica e retinopatia.

mente em pacientes com opacidade da córnea. Espera-se que, com estas iniciativas e com a ampliação dos conhecimentos sobre as manifestações oftalmológicas das MPS, haja uma melhora no perfil destes pacientes com relação ao aspecto da visão.

\section{REFERÊNCIAS}

1. Ashworth JL, Biswas S, Wraith E, Lloyd IC. Mucopolysaccharidoses and the eye. Surv Ophthalmol. 2006;51(1):1-17. Review

2. Willoughby CE, Ponzin D, Ferrari S, Lobo A, Landau K, Omidi Y. Anatomy and physiology of the human eye: effects of mucopolysaccharidoses disease on structure and function a review. Clin Exp Ophthalmol [Internet]. 2010 [cited 2010 Nov 20];38 2-11. Available from: http://onlinelibrary.wiley.com/doi/10.1111/j.1442-9071.2010.02363.x/pdf

3. Martin R, Beck M, Eng C, Giugliani R, Harmatz P, Muñoz V, Muenzer J. Recognition and diagnosis of mucopolysaccharidosis II (Hunter syndrome). Pediatrics. 2008;121(2): p. e377-86

4. Poupetova H, Ledvinová J, Berná L, Dvoráková L, Kozich V, Elleder M. The birth prevalence of lysosomal storage disorders in the Czech Republic: comparison with data in different populations. J Inherit Metab Dis. 2010;33(4):387-96.

5. Baehner F, Schmiedeskamp C, Krummenauer F, Miebach E, Bajbouj M, Whybra C, et al. Cumulative incidence rates of the mucopolysaccharidoses in Germany. J Inherit Metab Dis. 2005;28(6):1011-7.

6. Ashworth JL, Biswas S, Wraith E, Lloyd IC. The ocular features of the mucopolysaccharidoses. Eye (Lond., 2006;20(5):553-63.

7. Ferrari S, Ponzin D, Ashworth JL, Fahnehjelm KT, Summers CG, Harmatz PR, Scarpa M, et al. Diagnosis and management of ophthalmological features in patients with mucopolysaccharidosis. Br J Ophthalmol. 2011;95(5):613-9.

8. Wraith JE. Enzyme replacement therapy for the management of the mucopolysaccharidoses. Int J Clin Pharmacol Ther. 2009;47 Suppl 1: S63-5.

9. Decker C, Yu F, Giugliani R, Schwartz IV, Guffon N, Teles ET, et al. Enzyme replacement therapy for mucopolysaccharidosis VI: Growth and pubertal development in patients treated with recombinant human $\mathrm{N}$-acetylgalactosamine 4-sulfatase. J Pediatr Rehabil Med. 2010;3(2):89-100

10. Kakkis ED. Enzyme replacement therapy for the mucopolysaccharide storage disorders Expert Opin Investig Drugs. 2002;11(5):675-85.

11. Vieira T, Schwartz I, Muñoz V, Pinto L, Steiner C, Ribeiro M, et al., Mucopolysaccharidoses in Brazil: what happens from birth to biochemical diagnosis? Am J Med Genet A. 2008; 146A(13):1741-7.

12. Amorim, T., et al., Genetics in the "Sertão": Study of frequent monogenetic disorders in Monte Santo - a small city in the state of Bahia - northeastern Brazil. Acta Bioq Latinoamer. 2007;194(supl 1).

13. Fahnehjelm KT, Tornquist AL, Malm G, Winiarski J. Ocular findings in four children with mucopolysaccharidosis I-Hurler (MPS I-H) treated early with haematopoietic stem cell transplantation. Acta Ophthalmol Scand. 2006;84(6):781-5.

14. Fahnehjelm KT, Tornquist AL, WIniarski J. Ocular axial length and corneal refraction in children with mucopolysaccharidosis (MPS I-Hurler). Acta Ophthalmol. 2010. [Epub ahead of print]

15. Kara-José N, Rodrigues MD, editores. Saúde ocular e prevenção da cegueira. Cultura Médica: Rio de Janeiro; 2009. p.442.

16. Goldberg MF, Duke JR. Ocular histopathology in Hunter's syndrome. Systemic mucopolysaccharidosis type II. Arch Ophthalmol. 1967;77(4):503-12.

17. Alroy J, Haskins M, Birk DE. Altered corneal stromal matrix organization is associated with mucopolysaccharidosis I, III and VI. Exp Eye Res. 1999;68(5):523-30.

18. Ashworth $\mathrm{J}$, Kruse FE, Bachmann B, Tormene AP, Suppiej A, Parini R, et al. Ocular manifestations in the mucopolysaccharidoses - a review. Clin Exp Ophthalmol [Internet]. 2010 [cited 2010 Nov 20]; 38 Suppl 1:12-22. Available from: http://onlinelibrary.wiley.com/doi/ 10.1111/j.1442-9071.2010.02364.x/pdf

19. Mullaney P, Awad AH, Millar L. Glaucoma in mucopolysaccharidosis 1-H/S. J Pediatr Ophthalmol Strabismus. 1996;33(2):127-31.

20. Quigley HA, Maumenee AE, StarkWJ. Acute glaucoma in systemic mucopolysaccharidosis I-S. Am J Ophthalmol. 1975;80(1):70-2.

21. Delleman JW, de Jong PT. Pigment epithelial pattern dystrophy: a peripheral type. Br J Ophthalmol. 1985;69(10):754-7 